\title{
Uncoupled Dynamics Do Not Lead to Nash Equilibrium
}

\author{
By Sergiu Hart and Andreu Mas-Colell*
}

It is notoriously difficult to formulate sensible adaptive dynamics that guarantee convergence to Nash equilibrium. In fact, short of variants of exhaustive search (deterministic or stochastic), there are no general results; of course, there are many important, interesting and well-studied particular cases. See the books of Jörgen W. Weibull (1995), Fernando VegaRedondo (1996), Larry Samuelson (1997), Drew Fudenberg and David K. Levine (1998), Josef Hofbauer and Karl Sigmund (1998), H. Peyton Young (1998), and the discussion in Section IV below.

Here we provide a simple answer to the question: Why is that so? Our answer is that the lack of a general result is an intrinsic consequence of the natural requirement that dynamics of play be "uncoupled" among the players, that is, the adjustment of a player's strategy does not depend on the payoff functions (or utility functions) of the other players (it may depend on the other players' strategies, as well as on the payoff function of the player himself). This is a basic informational condition for dynamics of the "adaptive" or "behavioral" type.

It is important to emphasize that, unlike the existing literature (see Section IV), we make no "rationality" assumptions: our dynamics are not best-reply dynamics, or better-reply, or payoffimproving, or monotonic, and so on. What we show is that the impossibility result is due only

\footnotetext{
* Hart: Center for Rationality and Interactive Decision Theory, Department of Mathematics, and Department of Economics, The Hebrew University of Jerusalem, Feldman Building, Givat-Ram, 91904 Jerusalem, Israel (e-mail: hart@huji.ac.il; URL: 〈http://www.ma.huji.ac.il/hart〉); Mas-Colell: Department of Economics and Business, Universitat Pompeu Fabra, Ramon Trias Fargas 25-27, 08005 Barcelona, Spain (e-mail: mcolell@upf.es). The research is partially supported by grants of the Israel Academy of Sciences and Humanities, the Spanish Ministry of Education, the Generalitat de Catalunya, and the EU-TMR Research Network. We thank Robert J. Aumann, Yaacov Bergman, Vincent Crawford, Josef Hofbauer, Piero La Mura, Eric Maskin, Motty Perry, Alexander Vasin, Bob Wilson, and the referees for their useful comments.
}

to an "informational" requirement - that the dynamics be uncoupled.

\section{The Model}

The setting is that of games in strategic (or normal) form. Such a game $\Gamma$ is given by a finite set of players $N$, and, for each player $i \in N$, a strategy set $S^{i}$ (not necessarily finite) and a payoff function ${ }^{1} u^{i}: \prod_{j \in N} S^{j} \uparrow \mathbb{R}$.

We examine differential dynamical systems defined on a convex domain $X$, which will be either $\prod_{i \in N} S^{i}$ or $^{2} \prod_{i \in N} \Delta\left(S^{i}\right)$, and are of the form

$$
\dot{x}(t)=F(x(t) ; \Gamma),
$$

or $\dot{x}=F(x ; \Gamma)$ for short. We also write this as $\dot{x}^{i}=F^{i}(x ; \Gamma)$ for all $i$, where $x=\left(x^{i}\right)_{i \in N}$ and $^{3}$ $F=\left(F^{i}\right)_{i \in N}$.

From now on we keep $N$ and $\left(S^{i}\right)_{i \in N}$ fixed, and identify a game $\Gamma$ with its $N$-tuple of payoff functions $\left(u^{i}\right)_{i \in N}$, and a family of games with a set $\mathcal{U}$ of such $N$-tuples; the dynamics are thus

$$
\dot{x}^{i}=F^{i}\left(x ;\left(u^{j}\right)_{j \in N}\right) \text { for all } i \in N .
$$

We consider families of games $\mathcal{U}$ where every game $\Gamma \in \mathcal{U}$ has a single Nash equilibrium $\bar{x}(\Gamma)$. Such families are the most likely to allow for well-behaved dynamics. For example, the dynamic $\dot{x}=\bar{x}(\Gamma)-x$ will guarantee convergence to the Nash equilibrium starting from any

\footnotetext{
${ }^{1} \mathbb{R}$ denotes the real line.

${ }^{2}$ We write $\Delta(A)$ for the set of probability measures over $A$.

${ }^{3}$ For a well-studied example (see for instance Hofbauer and Sigmund, 1998), consider the class of "fictitious play"like dynamics: the strategy $q^{i}(t)$ played by $i$ at time $t$ is some sort of "good reply" to the past play of the other players $j$, i.e., to the time average $x^{j}(t)$ of $q^{j}(\tau)$ for $\tau \leq t$; then (after rescaling the time axis) $\dot{x}^{i}=q^{i}-x^{i} \equiv G^{i}(x ; \Gamma)-x^{i} \equiv$ $F^{i}(x ; \Gamma)$.
} 
initial condition. ${ }^{4}$ Note, however, that in this dynamic $\dot{x}^{i}$ depends on $\bar{x}^{i}(\Gamma)$, which, in turn, depends on all the components of the game $\Gamma$, in particular on $u^{j}$ for $j \neq i$. This motivates our next definition.

We call a dynamical system $F(x ; \Gamma)$ (defined for $\Gamma$ in a family of games $\mathcal{U}$ ) uncoupled if, for every player $i \in N$, the function $F^{i}$ does not depend on $u^{j}$ for $j \neq i$; i.e.,

$$
\dot{x}^{i}=F^{i}\left(x ; u^{i}\right) \text { for all } i \in N
$$

[compare with (1)]. Thus the change in player $i$ 's strategy can be a function of the current $N$-tuple of strategies $x$ and $i$ 's payoff function $u^{i}$ only. ${ }^{5}$ In other words, if the payoff function of player $i$ is identical in two games in the family, then at each $x$ his strategy $x^{i}$ will change in the same way. ${ }^{6}$

If, given a family $\mathcal{U}$ with the single-Nashequilibrium property, the dynamical system always converges to the unique Nash equilibrium of the game for any game $\Gamma \in \mathcal{U}$-i.e., if $F(\bar{x}(\Gamma) ; \Gamma)=0$ and $\lim _{t \uparrow \infty} x(t)=\bar{x}(\Gamma)$ for any solution $x(t)$ (with any initial condition) - then we will call $F$ a Nash-convergent dynamic for $\mathcal{U}$. To facilitate the analysis, we always restrict ourselves to $C^{1}$ functions $F$ with the additional property that at the (unique) rest point $\bar{x}(\Gamma)$ the Jacobian matrix $J$ of $F(\cdot ; \Gamma)$ is hyperbolic and (asymptotically) stable-i.e., all eigenvalues of $J$ have negative real parts.

We will show that:

\section{There exist no uncoupled dynamics which guarantee Nash convergence.}

Indeed, in the next two sections we present two simple families of games (each game having a single Nash equilibrium), for which uncoupledness and Nash convergence are mutually incompatible.

More precisely, in each of the two cases we exhibit a game $\Gamma_{0}$ and show that: ${ }^{7}$

\footnotetext{
${ }^{4}$ The same applies to various generalized Newton methods and fixed-point-convergent dynamics.

${ }^{5}$ It may depend on the function $u^{i}(\cdot)$, not just on the current payoffs $u^{i}(x)$.

${ }^{6}$ What the other players $d o$ (i.e., $x^{-i}$ ) is much easier to observe than why they do it (i.e., their utility functions $u^{-i}$ ).

${ }^{7}$ An $\varepsilon$-neighborhood of a game $\Gamma_{\mathrm{O}}=\left(u_{\mathrm{O}}^{i}\right)_{i \in N}$ consists of all games $\Gamma=\left(u^{i}\right)_{i \in N}$ satisfying $\left|u^{i}(s)-u_{0}^{i}(s)\right|<\varepsilon$ for all $s \in \prod_{i \in N} S^{i}$ and all $i \in N$.
}

THEOREM 1: Let $\mathcal{U}$ be a family of games containing a neighborhood of the game $\Gamma_{0}$. Then every uncoupled dynamic for $\mathcal{U}$ is not Nash-convergent.

Thus an arbitrarily small neighborhood of $\Gamma_{0}$ is sufficient for the impossibility result (of course, nonexistence for a family $\mathcal{U}$ implies nonexistence for any larger family $\mathcal{U}^{\prime} \supset \mathcal{U}$ ).

\section{An Example with a Continuum of Strategies}

Take $N=\{1,2\}$ and $S^{1}=S^{2}=D$, where $D:=\left\{z=\left(z_{1}, z_{2}\right) \in \mathbb{R}^{2}:\|z\| \leq 1\right\}$ is the unit disk. Let $\phi: D \uparrow D$ be a continuous function that satisfies:

- $\phi(z)=2 z$ for $z$ in a neighborhood of 0 ; and - $\phi(\phi(z)) \neq z$ for all $z \neq 0$.

Such a function clearly exists; for instance, let us put $\phi(z)=2 z$ for all $\|z\| \leq 1 / 3$, define $\phi$ on the circle $\|z\|=1$ to be a rotation by, say, $\pi / 4$, and interpolate linearly on rays between $\|z\|=1 / 3$ and $\|z\|=1$.

Define the game $\Gamma_{0}$ with payoff functions $u_{0}^{1}$ and $u_{0}^{2}$ given by $^{8}$

$$
u_{0}^{i}\left(x^{i}, x^{j}\right):=-\left\|x^{i}-\phi\left(x^{j}\right)\right\|^{2} \text { for all } x^{i}, x^{j} \in D .
$$

$\Gamma_{0}$ has a unique Nash equilibrium ${ }^{9} \bar{x}=(0,0)$.

We embed $\Gamma_{0}$ in the family $\mathcal{U}_{0}$ consisting of all games $\Gamma \equiv\left(u^{1}, u^{2}\right)$ where, for each $i=1,2$, we have $u^{i}\left(x^{i}, x^{j}\right)=-\left\|x^{i}-\xi^{i}\left(x^{j}\right)\right\|^{2}$, with $\xi^{i}: D \uparrow \quad D$ a continuous function, such that the equation $\xi^{i}\left(\xi^{j}\left(x^{i}\right)\right)=x^{i}$ has a unique solution $\bar{x}^{i}$. Then $\bar{x}=\left(\bar{x}^{1}, \bar{x}^{2}\right)$ is the unique Nash equilibrium of the game ${ }^{10} \Gamma$.

We will now prove that every uncoupled dynamic for $\mathcal{U}_{0}$ is not Nash-convergent. This proof contains the essence of our argument, and

\footnotetext{
${ }^{8}$ We use $j:=3-i$ throughout this section. In the game $\Gamma_{\mathrm{o}}$, each player $i$ wants to choose $x^{i}$ so as to match as closely as possible a function of the other player's choice, namely, $\phi\left(x^{j}\right)$.

${ }^{9} \bar{x}$ is a pure Nash equilibrium if and only if $\bar{x}^{1}=\phi\left(\bar{x}^{2}\right)$ and $\bar{x}^{2}=\phi\left(\bar{x}^{1}\right)$, or $\bar{x}^{i}=\phi\left(\phi\left(\bar{x}^{i}\right)\right)$ for $i=1,2$. There are no mixed-strategy equilibria since the best reply of $i$ to any mixed strategy of $j$ is always unique and pure.

${ }^{10}$ Moreover $\bar{x}$ is a strict equilibrium.
} 
the technical modifications needed for obtaining Theorem 1 are relegated to the Appendix. Let $F$ thus be, by contradiction, a dynamic for the family $\mathcal{U}_{0}$ which is uncoupled and Nash-convergent. The dynamic can thus be written: $\dot{x}^{i}=$ $F^{i}\left(x^{i}, x^{j} ; u^{i}\right)$ for $i=1,2$.

The following key lemma uses uncoupledness repeatedly.

LEMMA 2: Assume that $y^{i}$ is the unique $u^{i}$ best-reply of $i$ to a given $y^{j}$, i.e., $u^{i}\left(y^{i}, y^{j}\right)>$ $u^{i}\left(x^{i}, y^{j}\right)$ for all $x^{i} \neq y^{i}$. Then $F^{i}\left(y^{i}, y^{j} ; u^{i}\right)=0$, and the eigenvalues of the $2 \times 2$ Jacobian matrix $^{11} J^{i}=\left(\partial F_{k}^{i}\left(y^{i}, y^{j} ; u^{i}\right) / \partial x_{l}^{i}\right)_{k, l=1,2}$ have negative real parts.

\section{PROOF:}

Let $\Gamma_{1}$ be the game $\left(u^{i}, u^{j}\right)$ with $u^{j}\left(x^{i}, x^{j}\right):=$ $-\left.\left\|x^{j}-y^{j}\right\|\right|^{2}$ (i.e., $\xi^{j}$ is the constant function $\left.\xi^{j}(z) \equiv y^{j}\right)$; then $\left(y^{i}, y^{j}\right)$ is its unique Nash equilibrium, and thus $F^{i}\left(y^{i}, y^{j} ; u^{i}\right)=0$. Apply this to player $j$, to get $F^{j}\left(x^{i}, y^{j} ; u^{j}\right)=0$ for all $x^{i}$ (since $y^{j}$ is the unique $u^{j}$-best-reply to any $x^{i}$ ). Hence $\partial F_{k}^{j}\left(x^{i}, y^{j} ; u^{j}\right) / \partial x_{l}^{i}=0$ for $k, l=1,2$. The $4 \times 4$ Jacobian matrix $J$ of $F\left(\cdot, \cdot ; \Gamma_{1}\right)$ at $\left(y^{i}\right.$, $\left.y^{j}\right)$ is therefore of the form

$$
J=\left[\begin{array}{ll}
J^{i} & K \\
0 & L
\end{array}\right] .
$$

The eigenvalues of $J$-which all have negative real parts by assumption-consist of the eigenvalues of $J^{i}$ together with the eigenvalues of $L$, and the result follows.

Put $f^{i}(x):=F^{i}\left(x ; u_{0}^{i}\right)$; Lemma 2 implies that the eigenvalues of the $2 \times 2$ Jacobian matrix $J^{i}:=\left(\partial f_{k}^{i}(0,0) / \partial x_{l}^{i}\right)_{k, l=1,2}$ have negative real parts. Again by Lemma $2, f^{i}\left(\phi\left(x^{j}\right), x^{j}\right)=0$ for all $x^{j}$, and therefore in particular $f^{i}\left(2 x^{j}, x^{j}\right)=0$ for all $x^{j}$ in a neighborhood of 0 . Differentiating and then evaluating at $\bar{x}=(0,0)$ gives

$2 \partial f_{k}^{i}(0,0) / \partial x_{l}^{i}+\partial f_{k}^{i}(0,0) / \partial x_{l}^{j}=0$

for all $k, l=1,2$.

\footnotetext{
${ }^{11}$ Subscripts denote coordinates: $x^{i}=\left(x_{1}^{i}, x_{2}^{i}\right)$ and $F^{i}=$ $\left(F_{1}^{i}, F_{2}^{i}\right)$.
}

Therefore the $4 \times 4$ Jacobian matrix $J$ of the system $\left(f^{1}, f^{2}\right)$ at $\bar{x}=(0,0)$ is

$$
J=\left[\begin{array}{cc}
J^{1} & -2 J^{1} \\
-2 J^{2} & J^{2}
\end{array}\right] .
$$

LEMMA 3: If the eigenvalues of $J^{1}$ and $J^{2}$ have negative real parts, then J has at least one eigenvalue with positive real part.

\section{PROOF:}

The coefficient $a_{3}$ of $\lambda$ in the characteristic polynomial $\operatorname{det}(J-\lambda I)$ of $J$ equals the negative of the sum of the four $3 \times 3$ principal minors; a straightforward computation shows that

$$
a_{3}=3 \operatorname{det}\left(J^{1}\right) \operatorname{trace}\left(J^{2}\right)+3 \operatorname{det}\left(J^{2}\right) \operatorname{trace}\left(J^{1}\right) .
$$

But $\operatorname{det}\left(J^{i}\right)>0$ and $\operatorname{trace}\left(J^{i}\right)<0$ (since the eigenvalues of $J^{i}$ have negative real parts), so that $a_{3}<0$.

Let $\lambda_{1}, \lambda_{2}, \lambda_{3}, \lambda_{4}$ be the eigenvalues of $J$. Then

$$
\begin{gathered}
\lambda_{1} \lambda_{2} \lambda_{3}+\lambda_{1} \lambda_{2} \lambda_{4}+\lambda_{1} \lambda_{3} \lambda_{4}+\lambda_{2} \lambda_{3} \lambda_{4} \\
=-a_{3}>0
\end{gathered}
$$

from which it follows that at least one $\lambda_{r}$ must have positive real part.

This shows that the unique Nash equilibrium $\bar{x}=(0,0)$ is unstable for $F\left(\cdot ; \Gamma_{0}\right)$ - a contradiction which establishes our claim.

For a suggestive illustration, ${ }^{12}$ see Figure 1 , which is drawn for $x$ in a neighborhood of $(0,0)$ where $\phi\left(x^{i}\right)=2 x^{i}$. In the region $\left\|x^{2}\right\| / 2<\left\|x^{1}\right\|<$ $2\left\|x^{2}\right\|$ the dynamic leads "away" from $(0,0)$ (the arrows show that, for $x^{j}$ fixed, the dynamic on $x^{i}$ must converge to $x^{i}=2 x^{j}$ - see Lemma 2).

\section{An Example with Finitely Many Strategies}

If the games have a finite number of strategies (i.e., if the $S^{i}$ are finite), then the state space for the dynamics is the space of $N$-tuples of mixed strategies $\prod_{i \in N} \Delta\left(S^{i}\right)$.

\footnotetext{
${ }^{12}$ The actual dynamic is 4-dimensional and may be quite complex.
} 


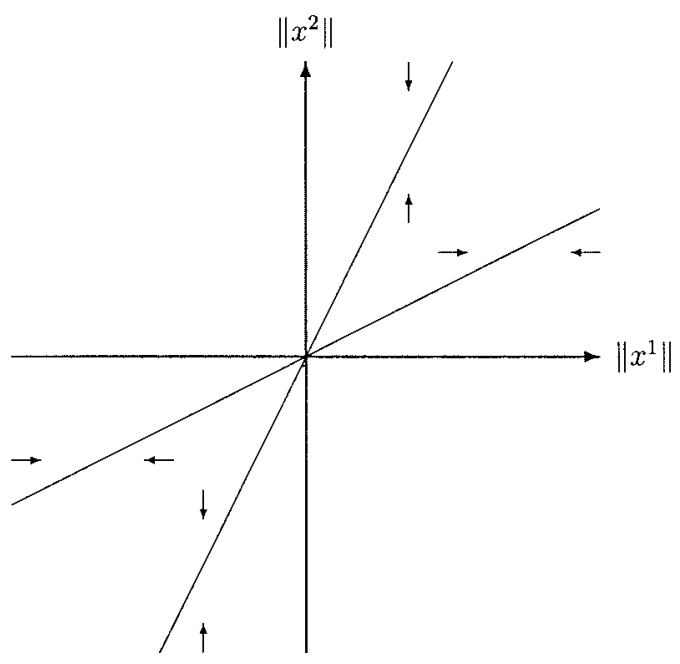

Figure 1. The Dynamic for the Game $\Gamma_{0}$ of Section II Around $(0,0)$

Consider a family $\mathcal{U}_{0}$ of three-player games where each player has two strategies, and the payoffs are:

\begin{tabular}{|l|l|}
\hline $0,0,0$ & $a^{1}, 1,0$ \\
\hline $1,0, a^{3}$ & $0,1, a^{3}$ \\
\hline
\end{tabular}

\begin{tabular}{|l|l|}
\hline $0, a^{2}, 1$ & $a^{1}, 0,1$ \\
\hline $1, a^{2}, 0$ & $0,0,0$ \\
\hline
\end{tabular}

where all the $a^{i}$ are close to 1 (say, $1-\varepsilon<a^{i}<$ $1+\varepsilon$ for some small $\varepsilon>0$ ), and, as usual, player 1 chooses the row, player 2 the column and player 3 the matrix. ${ }^{13}$ Let $\Gamma_{0}$ be the game with $a^{i}=1$ for all $i$; this game has been introduced by James Jordan (1993).

Denote by $x^{1}, x^{2}, x^{3} \in[0,1]$ the probability of the top row, left column, and left matrix, respectively. For every game in the family $\mathcal{U}_{0}$ there is a unique Nash equilibrium: ${ }^{14} \bar{x}^{i}(\Gamma)=$ $a^{i-1} /\left(a^{i-1}+1\right)$. In particular, for Jordan's game $\bar{x}_{\mathrm{O}} \equiv \bar{x}\left(\Gamma_{\mathrm{O}}\right)=(1 / 2,1 / 2,1 / 2)$.

Let $F$ be, by way of contradiction, an uncoupled

\footnotetext{
${ }^{13}$ Each player $i$ wants to mismatch the next player $i+1$, regardless of what player $i-1$ does. (Of course, $i \pm 1$ is always taken modulo 3 .)

${ }^{14}$ In equilibrium: if $i$ plays pure, then $i-1$ plays pure, so all players play pure-but there is no pure equilibrium; if $i$ plays completely mixed, then $a^{i}\left(1-x^{i+1}\right)=x^{i+1}$.
}

Nash-convergent dynamic $\mathcal{U}_{0}$. For the game $\Gamma_{0} \equiv$ $\left(u_{0}^{i}\right)_{i=1,2,3}$ we denote $f^{i}\left(x^{1}, x^{2}, x^{3}\right):=F^{i}\left(x^{1}, x^{2}, x^{3}\right.$; $u_{0}^{i}$ ); let $J$ be the $3 \times 3$ Jacobian matrix of $f$ at $\bar{x}_{0}$.

For any $y^{1}$ (close to $1 / 2$ ), the unique equilibrium of the game $\Gamma_{1}=\left(u_{0}^{1}, u_{0}^{2}, u^{3}\right)$ in $\mathcal{U}_{0}$ with $u^{3}$ given by $a^{3}=y^{1} /\left(1-y^{1}\right)$ is $\left(y^{1}, 1 / 2,1 / 2\right)$, and so $F^{1}\left(y^{1}, 1 / 2,1 / 2 ; \Gamma_{1}\right)=0$. This holds therefore also for $\Gamma_{0}$ since the dynamic is uncoupled: $f^{1}\left(y^{1}, 1 / 2,1 / 2\right)=0$ for all $y^{1}$ close to $1 / 2$. Hence $\partial f^{1}\left(\bar{x}_{0}\right) / \partial x^{1}=0$. The same applies to the other two players, and we conclude that the diagonal - and thus the trace- of the Jacobian matrix $J$ vanishes. Together with hyperbolicity [in fact, $\operatorname{det}(J) \neq 0$ suffices here], this implies the existence of an eigenvalue with positive real part, ${ }^{15}$ thus establishing our contradictionwhich proves Theorem 1 in this case.

We put on record that the uncoupledness of the dynamic implies additional structure on $J$. Indeed, we have $f^{1}\left(x^{1}, 1 / 2, x^{3}\right)=0$ for all $x^{1}$ and $x^{3}$ close to $1 / 2$ [since $\left(x^{1}, 1 / 2, x^{3}\right)$ is the unique Nash equilibrium when $a^{1}=1-$ as in $\Gamma_{0}$ - and $\left.a^{2}=x^{3} /\left(1-x^{3}\right), a^{3}=x^{1} /\left(1-x^{1}\right)\right]$. Therefore $\partial f^{1}\left(\bar{x}_{0}\right) / \partial x^{3}=0$ too, and so $J$ is of the form

$$
J=\left[\begin{array}{lll}
0 & c & 0 \\
0 & 0 & d \\
e & 0 & 0
\end{array}\right]
$$

for some real ${ }^{16} c, d, e$.

We conclude by observing that the specificities of the example have played very little role in the discussion. In particular, the property that the trace of the Jacobian matrix is null, or that $f^{i}$ vanishes over a linear subspace of co-dimension 1 , which is determined from the payoff function of player $i$ only, will be true for any uncoupled dynamics at the equilibrium of a game with a completely mixed Nash equilibrium-provided, of course, that the game is embedded in an appropriate family of games.

\footnotetext{
${ }^{15}$ Indeed: otherwise the real parts of all eigenvalues are 0 . The dimension being odd implies that there must be a real eigenvalue. Therefore 0 is an eigenvalue-and the determinant vanishes.

${ }^{16}$ If $c d e \neq 0$ there is an eigenvalue with positive part, and if $c d e=0$ then 0 is the only eigenvalue.
} 


\section{Discussion}

(a) There exist uncoupled dynamics converging to correlated equilibria ${ }^{17}$ - see Dean Foster and Rakesh V. Vohra (1997), Fudenberg and Levine (1999), Hart and MasColell (2000), ${ }^{18}$ and Hart and Mas-Colell (2003). It is thus interesting that Nash equilibrium, a notion that does not predicate coordinated behavior, cannot be guaranteed to be reached in an uncoupled way, while correlated equilibrium, a notion based on coordination, can. ${ }^{19}$

(b) In a general economic equilibrium framework, the parallel of Nash equilibrium is Walrasian (competitive) equilibrium. It is again well known that there are no dynamics that guarantee the general convergence of prices to equilibrium prices if the dynamic has to satisfy natural uncoupledness-like conditions, for example, the nondependence of the adjustment of the price of one commodity on the conditions of the markets for other commodities (see Donald G. Saari and Carl P. Simon, 1978).

(c) In a mechanism-design framework, the counterpart of the uncoupledness condition is Leonid Hurwicz's "privacy-preserving" or "decentralized" condition-see Hurwicz (1986).

(d) There are various results in the literature, starting with Lloyd S. Shapley (1964, Sec. 5 ), showing that certain classes of dynamics cannot be Nash-convergent. These dynamics assume that the players adjust to the current state $x(t)$ in a way that is, roughly speaking, payoff-improving; this includes fictitious play, best-reply dynamics, betterreply dynamics, monotonic dynamics, adjustment dynamics, replicator dynamics, and so on; see Vincent P. Crawford (1985), Jordan (1993), Andrea Gaunesdorfer and

\footnotetext{
${ }^{17}$ Of course, these dynamics are defined on the appropriate state space of joint distributions $\Delta\left(\Pi_{i \in N} S^{i}\right)$, i.e., probability vectors on $\mathrm{N}$-tuples of (pure) strategies.

${ }^{18}$ In fact, the notion of "decoupling" appears in Section 4 (i) there.

${ }^{19}$ Cum grano salis this may be called the "Coordination Conservation Law": there must be some coordination either in the equilibrium concept or in the dynamic.
}

Hofbauer (1995), Foster and Young (1998, 2001), and Hofbauer and Sigmund (1998, Theorem 8.6.1). All these dynamics are necessarily uncoupled (since a player's "good reply" to $x(t)$ depends only on his own payoff function). Our result shows that what underlies such impossibility results is not necessarily the rationality-type assumptions on the behavior of the players-but rather the informational requirement of uncoupledness.

(e) In a two-population evolutionary context, Alexander Vasin (1999) shows that dynamics that depend only on the vector of payoffs of each pure strategy against the current state-a special class of uncoupled dynamics-cannot be Nash-convergent.

(f) There exist uncoupled dynamics that are guaranteed to converge to (the set of) Nash equilibria for special families of games, like two-person zero-sum games, two-person potential games, dominance-solvable games, and others; ${ }^{20}$ for some recent work see Hofbauer and William H. Sandholm (2002) and Hart and Mas-Colell (2003).

(g) There exist uncoupled dynamics that are most of the time close to Nash equilibria, but are not Nash-convergent (they exit infinitely often any neighborhood of Nash equilibria); see Foster and Young (2002).

(h) Sufficient epistemic conditions for Nash equilibrium-see Robert J. Aumann and Adam Brandenburger (1995, Preliminary Observation, p. 1161) —are for each player $i$ to know the $N$-tuple of strategies $x$ and his own payoff function $u^{i}$. But that is precisely the information a player uses in an uncoupled dynamic-which we have shown not to yield Nash equilibrium. This points out the difference between the static and the dynamic frameworks: converging to equilibrium is a more stringent requirement than being in equilibrium.

(i) By their nature, differential equations allow strategies to be conditioned only on the limited information of the past captured by

\footnotetext{
${ }^{20}$ A special family of games may be thought of as giving information on the other players' payoff function (e.g., in two-person zero-sum games and potential games, $u^{i}$ of one player determines $u^{j}$ of the other player).
} 
the state variable. It may thus be of interest to investigate the topic of this paper in more general settings.

\section{APPENDIX}

We show here how to modify the argument of Section II in order to prove Theorem 1. Consider a family of games $\mathcal{U}$ that is a neighborhood of $\Gamma_{0}$, and thus is certain to contain only those games in $\mathcal{U}_{0}$ that are close to $\Gamma_{0}$. The proof of Lemma 2 uses payoff functions of the form $u^{j}\left(x^{i}, x^{j}\right)=-\left\|x^{j}-y^{j}\right\|^{2}$ that do not depend on the other player's strategy $x^{i}$ (i.e., $\xi^{j}$ is the constant function $\left.\xi^{j}(z) \equiv y^{j}\right)$. Since the proof in Section II needs the result of Lemma 2 only for $y^{j}$ in a neighborhood of 0 , we will replace the above constant function $\xi^{j}$ with a function that is constant in a neighborhood of the origin and is close to $\phi$.

We will thus construct for each $a \in D$ with $\|a\|<\varepsilon$ a function $\psi_{a}: D \uparrow \quad D$ such that: (1) $\left\|\psi_{a}-\phi\right\| \leq C \varepsilon$ for some constant $C>0$; (2) $\psi_{a}(z)=a$ for all $\|z\| \leq 2 \varepsilon$; (3) $\phi\left(\psi_{a}(z)\right)=z$ if and only if $z=\phi(a)=2 a$; and (4) $\psi_{b}\left(\psi_{a}(z)\right)=$ $z$ if and only if $z=\psi_{b}(a)=b$. The games corresponding to $\left(\phi, \psi_{y^{j}}\right)$ and to $\left(\psi_{x^{i}}, \psi_{y^{j}}\right)$, for $\left\|y^{j}\right\|<\varepsilon / 2$ and $x^{i}$ close to $2 y^{j}$, are therefore in $\mathcal{U}_{0}$ [by (3) and (4)], are close to $\Gamma_{0}[$ by (1)], and we can use them to obtain the result of Lemma 2 [by (2)].

The $\psi$ functions may be constructed as follows: (i) $\psi_{a}(z):=a$ for $\|z\| \leq 2 \varepsilon$; (ii) $\psi_{a}(z):=$ 0 for $\|z\|=3 \varepsilon$; (iii) $\psi_{a}(z)$ is a rotation of $\phi(z)$ by the angle $\varepsilon$ for $\|z\| \geq 4 \varepsilon$; and (iv) interpolate linearly on rays in each one of the two regions $2 \varepsilon<\|z\|<3 \varepsilon$ and $3 \varepsilon<\|z\|<4 \varepsilon$.

It can be checked that conditions (1)-(4) above are indeed satisfied. ${ }^{21}$

\footnotetext{
21 (1) and (2) are immediate. For (3), let $z=\phi(w)$ and $w=\psi_{a}(z)$. If $\|z\| \leq 3 \varepsilon$, then $w=\alpha a$ for some $\alpha \in[0,1]$, therefore $\|w\|<\varepsilon$ and $z=\phi(w)=2 w$, so in fact $\|z\|<2 \varepsilon$ and $w=a$. If $\|z\|>3 \varepsilon$, then, denoting by $\theta(x)$ the angle of $x$ and recalling that $\phi$ rotates by an angle between 0 and $\pi / 4$, we have $\theta(z)-\theta(w)=\theta(\phi(w))-\theta(w) \in[0, \pi / 4]$, whereas $\theta(w)-\theta(z)=\theta\left(\psi_{a}(z)\right)-\theta(z)=\theta(\phi(z))+\varepsilon-$ $\theta(z) \in[\varepsilon, \pi / 4+\varepsilon]$, a contradiction. (4) is proven in a similar way.
}

\section{REFERENCES}

Aumann, Robert J. and Brandenburger, Adam. "Epistemic Conditions for Nash Equilibrium." Econometrica, September 1995, 63(5), pp. 1161-80.

Crawford, Vincent P. "Learning Behavior and Mixed-Strategy Nash Equilibrium." Journal of Economic Behavior and Organization, March 1985, 6(1), pp. 69-78.

Foster, Dean and Vohra, Rakesh V. "Calibrated Learning and Correlated Equilibrium." Games and Economic Behavior, October 1997, 21(1), pp. 40-55.

Foster, Dean and Young, H. Peyton. "On the Non-Convergence of Fictitious Play in Coordination Games." Games and Economic Behavior, October 1998, 25(1), pp. 79-96.

. "On the Impossibility of Predicting the Behavior of Rational Agents." Proceedings of the National Academy of Sciences, October 2001, 98(22), pp. 12848-53.

"Learning, Hypothesis Testing, and Nash Equilibrium." Mimeo, Johns Hopkins University, 2002.

Fudenberg, Drew and Levine, David K. Theory of learning in games. Cambridge, MA: MIT Press, 1998.

. "Conditional Universal Consistency." Games and Economic Behavior, October 1999, 29(1), pp. 104-30.

Gaunesdorfer, Andrea and Hofbauer, Josef. "Fictitious Play, Shapley Polygons, and the Replicator Equation." Games and Economic Behavior, November 1995, 11(2), pp. 279303.

Hart, Sergiu and Mas-Colell, Andreu. "A Simple Adaptive Procedure Leading to Correlated Equilibrium." Econometrica, September 2000, 68(5), pp. 1127-50.

. "Regret-Based Continuous-Time Dynamics." Games and Economic Behavior, January 2003, 45(2), pp. 375-94.

Hofbauer, Josef and Sandholm, William H. "On the Global Convergence of Stochastic Fictitious Play." Econometrica, November 2002, 70(6), pp. 2265-94.

Hofbauer, Josef and Sigmund, Karl. Evolutionary games and population dynamics. Cambridge: Cambridge University Press, 1998. 
Hurwicz, Leonid. "Incentive Aspects of Decentralization," in K. J. Arrow and M. D. Intriligator, eds., Handbook of mathematical economics, Vol. III. Amsterdam: North-Holland, 1986, Ch. 28, pp. 1441-82.

Jordan, James. "Three Problems in Learning Mixed-Strategy Nash Equilibria." Games and Economic Behavior, July 1993, 5(3), pp. 368-86.

Saari, Donald G. and Simon, Carl P. "Effective Price Mechanisms." Econometrica, September 1978, 46(5), pp. 1097-125.

Samuelson, Larry. Evolutionary games and equilibrium selection. Cambridge, MA: MIT Press, 1997.

Shapley, Lloyd S. "Some Topics in Two-Person
Games," in M. Dresher, L. S. Shapley, and A. W. Tucker, eds., Advances in game theory (Annals of Mathematics Studies 52). Princeton, NJ: Princeton University Press, 1964, pp. $1-28$.

Vasin, Alexander. "On Stability of Mixed Equilibria." Nonlinear Analysis, December 1999, 38(6), pp. 793-802.

Vega-Redondo, Fernando. Evolution, games, and economic behavior. New York: Oxford University Press, 1996.

Weibull, Jörgen W. Evolutionary game theory. Cambridge, MA: MIT Press, 1995.

Young, H. Peyton. Individual strategy and social structure. Princeton, NJ: Princeton University Press, 1998. 